\title{
Asthma phenotypes in childhood: conceptual thoughts on stability and transition
}

\author{
Ben Daniel Spycher and Claudia Elisabeth Kuehni
}

Affiliation: Paediatric Epidemiology, Institute of Social and Preventive Medicine, University of Bern, Bern, Switzerland.

Correspondence: Ben Daniel Spycher, Institute of Social and Preventive Medicine, University of Bern, Finkenhubelweg 11, CH-3012, Bern, Switzerland. E-mail: ben.spycher囚ispm.unibe.ch

0 @ERSpublications

The potential of latent transition analysis of asthma phenotypes will be seen larger cohort studies http://ow.ly/VUzAH

We have known for a long time that asthma-like symptoms in the first years of life predict later asthma poorly. Many preschool wheezers become asymptomatic by school age. A few will develop classic atopic asthma, which is likely to persist into later childhood and even adulthood, but the large majority of preschool children with asthma-like symptoms are difficult place into clear-cut categories. The clinical picture varies from child to child, and remissions and relapses are common. During past decades, many attempts have been made to define asthma phenotypes. Initial attempts were simple and based on a combination of expert opinion and observations made in early cohort studies [1]. More recently, researchers have used statistical clustering techniques, which should provide a more objective classification because they obtain phenotypes from observed data according to a predefined method. Most of us would agree that letting the data speak is a good idea. We want a classification that reflects true patterns of disease as they occur in the population. However, in spite of their promises, these methods are not entirely objective. They require investigators to make certain choices such as which variables to include or which age intervals to consider, and these choices matter.

Among all studies that have attempted to define childhood asthma phenotypes using clustering techniques, that by GARDEN et al. [2], in this issue of the European Respiratory Journal, makes perhaps the strongest claim to objectivity. This is because the authors used a particularly flexible model that avoids one of the critical decisions that other investigators have always needed to make (knowingly or not): how to deal with changes in the clinical picture over time. As children grow older, their symptoms will change. A child who wheezes only with respiratory infections during infancy, as they grow older, may have symptoms with physical exercise and when playing with pets, or may cease to have symptoms at all. 1) Are these changes part of the child's phenotype, i.e. the natural history of its particular disease; or 2) do they represent the transition from one phenotype to another (e.g. the transition from a nonatopic to an atopic phenotype)? Depending on which point of view is taken, different phenotypes will result.

1) According to the first viewpoint, phenotypes are traits that stick with a child much like the child's genotype does. The clinical manifestations of these phenotypes may, however, change over time, each phenotype having its distinct trajectory. Thus, previous investigations using repeated assessments of wheeze in the Avon Longitudinal Study on Parents and Children (ALSPAC) cohort and other birth cohorts identified distinct wheezing trajectories, which have been given labels such as "early persistent

Received: Dec 012015 | Accepted: Dec 032015

Support statement: B.D. Spycher was supported by a Swiss National Science Foundation fellowship (grant number PZ00P3_147987). Funding information for this article has been deposited with FundRef.

Conflict of interest: Disclosures can be found alongside the online version of this article at erj.ersjournals.com

Copyright OERS 2016 
wheeze", "early transient wheeze" or "late onset wheeze" [3-6]. Similarly we investigated symptoms of cough and wheeze at two time-points in the Leicestershire cohort studies and identified patterns such as "transient viral wheeze" and "atopic persistent wheeze" [7, 8]. In this model, membership of a given phenotype does not change as the child grows older, but rather, temporal changes in symptoms become characteristics of the phenotypes and are expressed in terms like "transient", "persistent" or "late onset" wheeze. It is, however, important to realise that the stability of phenotype membership is not a result of the data but was imposed onto the model by the investigators.

2) According to the second viewpoint, a phenotype is a characteristic mix of disease manifestations that can be used to classify children at any age. Children whose symptoms change with age and no longer fit this particular mix are, by definition, transitioning to another phenotype. For instance, a child with recurrent wheeze, atopic sensitisation and reversible airway narrowing will be assigned the phenotype "atopic asthma", regardless of age. If at a later time point, one or more of these manifestations is no longer present, the child will be assumed to have transitioned to another phenotype that better reflects the new manifestations.

Both these positions are extreme and the "truth", if we maintain that phenotypes reflect true underlying diseases and are not just convenient labels, is likely to lie between them. In fact, clustering studies that have used cross-sectional data [9-13] take a middle ground between these positions. These studies seek to identify phenotypes that optimally characterise the differences between children in a given age interval. Inevitably, different phenotypes will result for different age intervals (deviation from the second viewpoint) and, within the same cohort, children will not be classified into the same groups as they grow older but will be reshuffled into new groups (deviation from first viewpoint). For instance, cluster analyses in a clinical cohort from Paris identified a phenotype of "uncontrolled wheeze" at age <3 years and again at 5 years $[9,10]$. However, this phenotype was predominately nonatopic at younger age and predominately atopic at older age. Fewer than $40 \%$ of children with this phenotype at age $<3$ years tracked to the corresponding phenotype at 5 years, while the others transitioned to other phenotypes. The obvious disadvantage of separate analyses by age groups is that they ignore the children's history and the tendency of their conditions to track. We might ask, could we not tweak the phenotypes at each age slightly, so that they would show stronger tracking over time while at the same time providing a good representation of the differences at each age? This is precisely what the model used by GARDEN et al. [2] does.

In their study, GARDEN et al. [2] use cohort data to distinguish phenotypes that not only optimally characterise the prevailing differences between children at given ages but also allow for the tendency of conditions to track. This is indeed novel. They do this by fitting a latent transition model to data on a range of asthma manifestations measured at ages 1.5, 3, 5, 8 and 11.5 years in children from CAPS (Childhood Asthma Prevention Study) in Sydney, Australia. The great advantage of this model is that it is extremely flexible and can produce both extremes explained above plus any intermediate form. How does this work? At each age, a separate set of phenotypes is fitted to data from children of this age in a similar way as previous latent class analyses have done using cross-sectional data $[12,13]$. The difference, however, is that these cross-sectional sets of phenotypes are now connected over time. In the model, this connection is represented by transition probabilities, which are estimated for each possible transition between the phenotypes of subsequent ages. This is precisely the flexibility we need. In the first extreme, the model could, in principle, produce phenotypes that track perfectly, i.e. all children with a given phenotype at a given age neatly transition to only one phenotype at the next age. These succeeding phenotypes could differ in their combination of clinical manifestations, reflecting the changing clinical picture of the same group of children over time, and could thus be viewed as a single, perfectly tracking phenotype (first viewpoint). However, real-life data are likely to produce a model with some degree of reshuffling. During model fitting, all the sets of phenotypes and all transition probabilities are simultaneously estimated so as to best represent the data. The optimal model will consist of phenotypes that best characterise differences at each age and, at the same time, track strongly over time. Both of these aspects contribute to model fit and must be balanced against each other, as there is a trade-off between them. In the second extreme, the model can also be restricted to producing exactly the same phenotypes at each time-point (second viewpoint). This will, in general, reduce model fit compared to the optimal model (by reduced tracking and poorer representation of groups at each age). However, if the reduction in model fit is modest, the gains in practicality from having the same phenotype definitions for all ages may be worth the cost. Statistical model selection criteria can help make the choice.

So what did GARDEN et al. [2] find? The preferred model consisted of four phenotypes at each age. The characteristics of these phenotypes were constrained to be the same at 1.5-5 years (preschool) and at 8-11.5 years (school age) but could vary between these two broad age periods. However, differences between the two age periods were not substantial and are probably explained by differences in measurements, which, for the second period, included spirometry, airway hyperresponsiveness (AHR) and exhaled nitric oxide 
concentration (eNO). Essentially, the model distinguished an asymptomatic nonatopic phenotype, an asymptomatic atopic phenotype (both including some children with mild cough, wheeze or sneeze), a symptomatic nonatopic phenotype and a symptomatic atopic phenotype. Only the two symptomatic phenotypes qualify as "asthma-phenotypes". At all ages, the large majority of children in these two phenotypes reported asthma-related symptoms and over $90 \%$ used bronchodilators. In the atopic asthma phenotype, all children had positive skin-prick tests to at least one inhalant allergen. At school age, this group clearly had a classic asthma phenotype, characterised by poor lung function, AHR and eosinophilic inflammation ( $70 \%$ in the highest quintile of eNO). The nonatopic asthma group is more difficult to grasp: the proportions at school age with AHR, low forced expiratory volume in $1 \mathrm{~s}$ and high eNO differed little from the respective proportions in the asymptomatic groups. This phenotype was also the least stable over time, with $20-30 \%$ of the children transitioning to the asymptomatic nonatopic phenotype between successive time-points and about $10 \%$ transitioning to the atopic asthma phenotype between successive time-points up to 8 years. The atopic asthma phenotype, in contrast, was much more stable over time, with over $80 \%$ staying in the same group between successive time-points. While the nonatopic asthma phenotype decreased in prevalence from $35 \%$ at 1.5 years to $8 \%$ at 11.5 years, the atopic asthma phenotype increased from $2 \%$ to $19 \%$ over the same period.

How do these results match up with what we already know? In fact, they fit in neatly with many previous findings. To mention a few examples, the phenotypes "early transient wheeze" and "transient viral wheeze" described in the ALSPAC study and the Leicestershire cohort studies respectively $[3,7,8]$ may correspond to children starting in the nonatopic asthma group of this study and transitioning to the nonatopic asymptomatic group. Previously described intermediate- and late-onset asthma phenotypes [3, 4] are likely to be represented by the children transitioning from the asymptomatic atopic to the atopic asthma phenotype in the present study. Intermediate- and late-onset asthma phenotypes have been particularly strongly associated with atopy in earlier studies $[3,4,14]$. The present study would suggest that asthma is secondary to atopy in these children. Other studies have also shown a strong association between atopy and asthma persistence, as reflected by the atopic asthma group in the present study [5, 14]. Nonatopic asthma has resulted as a separate entity in some previous analyses [7, 9, 12]. In the two Leicestershire cohort studies, this entity was poorly replicated across the two cohorts [8], perhaps because it is characterised by a lot of transitions to the asymptomatic group, as the present study shows. What is new in the study by GARDEN et al. [2] is that the analysis approach they chose can neatly disentangle phenotypes from transition patterns in a way that is informed by the data.

The main weakness of the study by GARDEN et al. [2] is that it is based on a small $(\mathrm{n}=370)$ and selected study sample. While, as we have seen, the results accommodate many of the previous findings for preschool and schoolchildren, with only four phenotypes and the transitions between them, we are left with an uneasy feeling that there must be more to the story. For instance, who are the children with nonatopic asthma and what makes them wheeze? Are these children who respond to other environmental stimuli such as tobacco smoke or infections? Associations with parental smoking and older siblings suggest that this indeed might be the case. If so, do they not perhaps consist of further subgroups? The current sample was too small to allow estimating models with more than four phenotypes and so simply did not provide the resolution for finer details. So, while this study represents an important step in the right direction, the real potential of latent transition analyses waits to be seen in application to much larger cohort studies.

\section{References}

Spycher BD, Silverman M, Kuehni CE. Phenotypes of childhood asthma: are they real? Clin Exp Allergy 2010; 40: $1130-1141$.

2 Garden FL, Simpson JM, Mellis CM, et al. Change in the manifestations of asthma and asthma-related traits in childhood: a latent transition analysis. Eur Respir J 2016; 47: 499-509.

3 Henderson J, Granell R, Heron J, et al. Associations of wheezing phenotypes in the first 6 years of life with atopy, lung function and airway responsiveness in mid-childhood. Thorax 2008; 63: 974-980.

4 Savenije OE, Granell R, Caudri D, et al. Comparison of childhood wheezing phenotypes in 2 birth cohorts: ALSPAC and PIAMA. J Allergy Clin Immunol 2011; 127: 1505-1512 e1514.

5 Belgrave DC, Simpson A, Semic-Jusufagic A, et al. Joint modeling of parentally reported and physician-confirmed wheeze identifies children with persistent troublesome wheezing. J Allergy Clin Immunol 2013; 132: 575-583 e512.

6 Depner M, Fuchs O, Genuneit J, et al. Clinical and epidemiologic phenotypes of childhood asthma. Am J Respir Crit Care Med 2014; 189: 129-138.

7 Spycher BD, Silverman M, Brooke AM, et al. Distinguishing phenotypes of childhood wheeze and cough using latent class analysis. Eur Respir J 2008; 31: 974-981.

8 Spycher B, Silverman M, Pescatore AM, et al. Comparison of phenotypes of childhood wheeze and cough in 2 independent cohorts. J Allergy Clin Immunol 2013; 132: 1058-1067.

9 Just J, Gouvis-Echraghi R, Couderc R, et al. Novel severe wheezy young children phenotypes: boys atopic multiple-trigger and girls nonatopic uncontrolled wheeze. J Allergy Clin Immunol 2012; 130: 103-110. 
10 Just J, Saint-Pierre P, Gouvis-Echraghi R, et al. Wheeze phenotypes in young children have different courses during the preschool period. Ann Allergy Asthma Immunol 2013; 111: 256-261 e251.

11 Just J, Gouvis-Echraghi R, Rouve S, et al. Two novel, severe asthma phenotypes identified during childhood using a clustering approach. Eur Respir J 2012; 40: 55-60.

12 Herr M, Just J, Nikasinovic L, et al. Risk factors and characteristics of respiratory and allergic phenotypes in early childhood. J Allergy Clin Immunol 2012; 130: 389-396.

13 Weinmayr G, Keller F, Kleiner A, et al. Asthma phenotypes identified by latent class analysis in the ISAAC phase II Spain study. Clin Exp Allergy 2013; 43: 223-232.

14 Collins SA, Pike KC, Inskip HM, et al. Validation of novel wheeze phenotypes using longitudinal airway function and atopic sensitization data in the first 6 years of life: evidence from the Southampton Women's survey. Pediatr Pulmonol 2013; 48: 683-692. 